\title{
Review
}

\section{Diversity and Recruitment of Minorities in Clinical Trials: An Unmet Need}

\author{
Nathalie Vives, BsN, MBA, PMP* \\ Founder, IntCom Trials and Research, Avenue Nouvelle 78, Brussels, Belgium \\ "Corresponding author \\ Nathalie Vives, BsN, MBA, PMP \\ Founder, IntCom Trials and Research,Avenue Nouvelle 78, Brussels, Belgium; E-mail: nathalie.vives@intcomtrials.com
}

\section{Article information}

Received: April 24 $4^{\text {th }}, 2020$; Revised: May 24 $4^{\text {th }}, 2020$; Accepted: June 5 ${ }^{\text {th }}, 2020$; Published: June $16^{\text {th }}, 2020$

\section{Cite this article}

Vives N. Diversity and recruitment of minorities in clinical trials:An unmet need. Clin Trial Pract Open J. 2020; 3(I): 9-14. doi: I0.17/40/CTPOJ-3-II2

\section{ABSTRACT}

Patient centricity has led to the idea that drugs should answer medical needs not only defined by the medical bodies but relevant for the patients themselves. This implies that the diversity of the patients should be represented in clinical trials. Disparities in representation of diverse populations in clinical trials have been well-documented for several pathologies like arterial hypertension, diabetes but also in oncology. Today, Caucasian patients included in clinical trials represent at least $75 \%$ of the clinical trials population even in pathologies where minorities are largely more affected than Caucasians. Filling the gap in diversity is an important matter to ensure ethics, good practices, regulatory compliance, patient safety and scientific validity. To achieve their recruitment goals and being patient centric, sponsors and investigational sites need to pay attention of this problem and put in place specific strategies. Mistrust of patients of minority groups toward clinical research is one of the main barriers along with lack of access to clinical trials (low awareness, communication barriers, distance to investigational sites). Adapted communication, community-based strategies are some of the useful strategies directed to the patients. Investigators may not encounter diverse populations in their area or may not be aware of unconscious bias when identifying patients suitable for a clinical trial. Looking for referrals and raising awareness of the risk of bias with training on culturally sounded approaches could help investigators to recruit more diverse populations. Lack of awareness and costs may prevent sponsors to focus on diversity but ethics, benefits of data acquisition and patient centricity should be enough motivators to modify this trend. Patients, investigators and sponsors have their own barriers to tackle the lack of diversity in clinical trials. The cultural background of all stakeholders is an important factor in those barriers. Only multidisciplinary approaches including human sciences would help to define patient recruitment strategies that would mitigate the current lack of diversity.

\section{Keywords}

Clinical trials; Patient recruitment; Diversity, Minorities.

\section{INTRODUCTION}

$\mathrm{I}^{\mathrm{n}}$ $\mathrm{n}$ the later years, the patients' status has started to evolved from a mere subject to a stakeholder in drug development, with a raise of patient centricity approaches. ${ }^{1,2}$ The expectations from patients, voiced today mainly by patient advocacy groups, need to be taken in account.

One of these expectations is the accessibility of the drugs or medical devices to the patients who may benefit from them and accessibility in a form, dosage and administration adapted to their needs.
For this, the patient population pooled in clinical trials needs to reflect the reality of the patients having the disease or condition.

Patient recruitment is influenced at all levels by the culture of the different stakeholders engaged in its process. While some elements, more pragmatic, are taken into account when planning a clinical trial (translation and customization of documents), the cultural factors that may affect patients and investigators, are barely considered. ${ }^{2}$ Nonetheless, this factor is of upmost importance if we want to achieve patient centricity and ensure efficacy and safety of drugs, in particular when recruiting culturally diverse populations. 
While ethnicity may be discussed for specific trials involving Japanese or Chinese subjects due to regulatory requirements, most of the literature discussing cultural diversity in patient recruitment in clinical trials originates from the USA and a wide literature is now available approaching the topic from different perspectives (scientific, sociological, legal, ethical, etc.).

Before describing the current panorama of patient recruitment and diversity in clinical trials, it seems important to clarify and explain the connection between terms.

Patient centricity has been defined as 'Putting the patient first in an open and sustained engagement of the patient to respectfully and compassionately achieve the best experience and outcome for that person and their family. ${ }^{3}$ In clinical trials, this would translate in patient involvement in protocol design and consistent efforts to decrease the burden during the study execution and in the patient recruitment process. It would be rational to assume that different cultural background would affect the experience of patients and thus, that patient centricity cannot be achieved without considering diversity. It is also worth to highlight that patient centricity and diversity considerations are important focus for a large number of medium and large biopharmaceutical companies.

Disparity in representation is defined in this article as the difference of the relative participation of a group in clinical trials related to the relative proportion of this group in the population having a disease or condition.

The use of diversity and minorities terms needs also to be clarified. While diversity focus on the inclusion of different types of people, ${ }^{4}$ minorities refers not only as a part of a population differing from others in some characteristics but also often subjected to differential treatment. ${ }^{4}$ As racial and ethnic disparities in healthcare access has been largely documented, the hypothesis that these disparities could affect clinical trials arena seems reasonable. The term minorities may be used in this article when these disparities need to be highlighted.

Lastly, patient recruitment processes and management are not scientific/medical topics and while certain evaluations can use the scientific methods, the human factor widely impacts. This article also aims to highlight the need to approach patient recruitment not only from a scientific but also from sociological, ethical and legal perspectives.

\section{DIVERSITY IN PATIENT RECRUITMENT:WHAT IS THE REALITY?}

The lack of diversity in clinical trials has been raised in several contexts and populations. ${ }^{2,5-13}$ This lack of inclusion in clinical trials impacts populations such as racial and ethnic minorities but also women, elderlies and patients with unique comorbidities. While safety concerns and difficulty to demonstrate efficacy of a drug when drug-drug interactions are likely to happen may drive the exclusion of elderlies and patients with multiple pathologies, the exclusion of minorities and women appears to be driven by other factors, that will be discussed later.

While women were initially excluded from clinical trials around 1980s, several policies have been put in place to promote their inclusion, notably in the USA. ${ }^{11,14-16}$

It is unclear if sex parity in participation in clinical trials has been achieved, ${ }^{17}$ nonetheless, the historical background of women inclusion in clinical trials with its regulations is useful in the discussion the inclusion of ethnic and racial groups in clinical trials.

In 2015, African-Americans represented $12 \%$ of the total U.S. population but represented less than $5 \%$ of clinical trial participants. Hispanics represented $16 \%$ of the total population but only $1 \%$ participated in clinical trials. ${ }^{2}$ These disparities still appear when compared with the population having a certain disease or condition. Blacks or African-Americans have a $28 \%$ higher cancer-specific mortality compared with whites ${ }^{9}$ but are still underrepresented in oncological clinical trials. Multiple myeloma incidence in the USA is twice as common among African-Americans as it is among other racial or ethnic groups, ${ }^{18}$ while the population included in the clinical trials for the three main treatments of multiple myeloma is at least $75 \%$ white. ${ }^{19}$

In $2014,86 \%$ of the participants to the global and local clinical trials which data were used by the Food and Drug Administration (FDA) to approve drugs, were white, a small decrease from 1997 when $92 \%$ of those were white. ${ }^{20}$

While the extend of the problem in clinical trials varies depending on the disease or conditions, there is wide consensus about the underrepresentation of minorities in late stage clinical trials. A quick look at the extensive literature and the FDA drug snapshots which show per approved drugs the population included per race/ethnic groups could confirm this. ${ }^{19}$

\section{Regulatory Toward Diversity in Clinical Trials}

The American FDA, after the National Institutes of Health Revitalization Act in 1993, defined recommendations in 2011 for women and minorities inclusion in clinical trials. ${ }^{10}$

The final rule entitled 'Presentation of Safety and Effectiveness Data for Certain Subgroups of the Population in Investigational New Drug Application Reports and New Drug Applications' was issued by the FDA in 1998. This regulation specifically states that New Drug Applications (NDA) must present safety and efficacy data for important populations, including sex, age, and racial subgroups. ${ }^{14}$ The FDA has the authority to refuse to file any NDA that does not analyse the safety and efficacy data appropriately. ${ }^{14}$

More recently, in 2017, the FDA Reauthorization Act 
mandated a new guidance ${ }^{13}$ to be defined, guidance still in public review today. This guidance does not establish legally enforceable responsibilities and are not supported globally by any specific recommendations from the International Council for Harmonisation of Technical Requirements for Pharmaceuticals for Human Use (ICH). The European Medicines Agency has not published either any recommendations on this respect.

\section{LACK OF DIVERSITY IN CLINICAL TRIALS: WHY IS THIS A PROBLEM?}

The lack of diversity in clinical trial population described above has several implications for patients, investigators and sponsors. ${ }^{21}$

\section{Ethics, Good Practices and Regulation Compliance}

The Declaration of Helsinki states in its article 13 that 'groups that are underrepresented in medical research should be provided appropriate access to participation in research'. ${ }^{22}$ Amongst the factors, developed further in this article and leading to this underrepresentation of minorities groups is the specific lack of awareness of clinical trials of these populations. While not legally enforceable, the ethical principles of the Declaration of Helsinki are widely accepted and followed by medical researchers. The lack of diversity in clinical trials population should therefore be questioned from an ethical point of view.

As mentioned previously, the ICH hasn't developed specific recommendations for inclusion of diverse background populations. Nonetheless, the ICH E9 (Statistical Principles for Clinical Trials) guideline requires that the study population should be representative of the target patient population. ${ }^{23}$ This guidance is in step 5, meaning that its content should have been implemented in the ICH countries (USA, Europe, Japan) and recommendations applied.

Finally, the FDA can refuse to file an NDA that does not analyse the safety and efficacy data appropriately, considering specific populations affected by a disease or condition the product is meant to treat or prevent. ${ }^{14}$

\section{Safety, Effectiveness and Lack of Scientific Evidence}

The scientific impact of patient recruitment can be found in several studies conducted in diverse ethnic groups on several conditions which have shown an impact on drug response and disease pathology in determined minorities. ${ }^{9}, 21,24-28$ As an example, the discovery of a genetic variant that was protective against breast cancer in Latinas would not have happened if these ethnic population had not been the focus of this research. ${ }^{24}$

Another example is the use of antihypertensive therapy. Current recommendations of treatment are derived from data from clinical trials in Caucasians populations, although this pathology outcomes represent significantly more risks to the black populations. ${ }^{29}$ Variability of response to the $\beta$-blockers (first line treatment) of this population and in other ethnic groups is well-known.
Nonetheless, as more than $85 \%$ of hypertensive patients require several drugs to achieve blood pressure control, studies on this variability for second- (or third) line treatments in ethnic groups are missing and are required to ensure a proper evidence-based medicine. ${ }^{30}$

\section{Limit to the Pool of Potential Participants in Cllinical TrialsI}

Delays in subject recruitment is one of the main reasons for not achieving trials goals for timelines and costs and they are mainly due to difficulty in recruiting suitable patients.

While the lack of diversity is an adverse effect of current practices in patient recruitment and not something looked for, focusing on including population of diverse background would increase the number of potentially eligible patients.

\section{No Patient Centricity without Diversity}

Putting the patients first and engaging them means designing clinical trials so their endpoints and objectives are of interest for the patient community. As disease perceptions and behaviours varies with the cultural background of each person, ${ }^{31}$ patient centricity needs to take in account all their diverse interests.

\section{Economical Exclusion}

Considerations of cultural diversity may be thought also as a question of economical inclusion. Cultural minorities are generally associated with low socioeconomical status and socioeconomic constraints have been described as barriers to recruitment. ${ }^{6}$

Those effects impact patients, investigators and sponsors at different levels. But most of these effects are under the responsibilities of the investigators and sponsors.

\section{BARRIERS AND STRATEGIES FOR RECRUITMENT OF DIVERSE POPULATIONS}

An important number of studies have been focusing on the barriers to recruitment and strategies of diverse populations. Barriers amongst subjects, site or investigators and sponsors are often intertwined and strategies to overcome them need to be considered transversally.

\section{Subjects Barriers}

- Mistrust to clinical research and healthcare system. ${ }^{5-7,9,32,33}$ Nonetheless, mistrust doesn't imply unwillingness and once communication barriers overcome and rapport built with investigators, those patients tend to volunteer for clinical research.

- Language barriers and non-adapted communication, in particular associated with complex informed consent forms and procedures $^{5-7,33}$

- Lack of awareness of clinical trials ${ }^{5}$ 
- Participants beliefs about health and health care may clash with the prevailing norms of the society they are in. ${ }^{6,7}$

- Access to investigational sites: investigational sites are often located in areas distant from where racial and ethnic minorities live. ${ }^{8}$

- Access to general healthcare system: Inequality of access to clinical trials reflects in part, the inequality of access to health for those minorities. ${ }^{10}$

Recruitment strategies involving adapted communication, raising clinical trials awareness with community approaches and ensuring representativity of diverse population in site personnel along with selecting sites in location with a high concentration of racial and ethnic minority patients ${ }^{6,8,13,33}$ have been described as successful to tackle these barriers.

\section{Investigators/Sites Barriers}

- Lack of cultural sensitivity and appropriate training would affect negatively the process of informed consent and retention. ${ }^{6}$

- Lack of researchers from minorities population: Several studies have highlighted that patients from diverse background would have a higher trust and confidence with physicians sharing the same cultural background. $6,7,13,32,33$

- Investigator bias and 'good patient' profile: Constraints of time, costs and resources may lead to the search of a profile of patients that would be perceived as meticulous, proactive, compliant and with strong social support network, limiting the burden linked to recruitment and retention. ${ }^{34}$ Mistrust among the communities and socioeconomical issues, often present in minorities, may affect the perception of these patients as compliant and having a strong support network. Probably also related to the lack of cultural sensitivity, this unconscious bias, by its nature, represents a complex barrier to tackle.

- Site location and limited human resources

Approaches aiming to promote cultural diversity both in clinical trials recruitment but also in site personnel recruitment and to raise awareness of possible bias, via communication, events and training seems appropriate to mitigate the site intrinsic barriers. Site selection taking in account the location of diverse populations ${ }^{13}$ and the use of referrals and community approaches by the sites are other strategies that can be implemented for specific clinical trials.

\section{Sponsors Barriers}

- Lack of awareness of issues related to poor diversity in clinical trials

- Perceived cost of adapted patient recruitment approaches

- Increased complexity of clinical trials management due to multiplicity and adaptation of recruitment approaches

- Lack of robustness of most research on patient recruitment strategies. Patient recruitment strategies are experimental and their efficacy are not guaranteed. Their results of can rarely be applied to other context. ${ }^{7,35}$

Wide communication around issues and benefits of di- versity in clinical trials should be driven by patient centricity groups within sponsors but also by regulatory agencies and well-known consortiums in order to impact current practices. Considering balanced costs of adapted patient recruitment approaches to benefits of data acquisition and ethics and regulation compliance would give another perspective prone to focus on this matter.

\section{CONCLUSION}

Diversity in clinical trials needs to be ensured for ethics reasons, safety of patients, science and social justice. The lack of diversity is not a recent problem and has been widely documented since 1990s. ${ }^{36}$ With the rise of patient centricity, it becomes urgent that sponsors, if they want to achieve their objective of putting the patient first, embrace diversity making it possible in clinical trials.

To understand and implement successful strategies of patient recruitment for clinical trials, the cultural background of all stakeholders needs to be understood globally and locally and those approaches should be directed not only to the patients but also to investigational sites and sponsors. While patients from diverse populations need to be the focus of specific strategies for awareness of clinical trials, sponsors and sites need to be made aware of their own potential bias in patient recruitment practices.

The impact of culture in the success of patient recruitment strategies makes difficult to envision an assessment based solely on pure scientific approaches. While the scientific method and the business administration it implies need to be applied more rigorously, patient recruitment needs also to be approached from a sociological, anthropological, psychological, and also historical and legal perspectives using their own methodologies.

\section{ACKNOWLEDGEMENTS}

The author would like to thank Mundeep Matharu, Jean-David Bienvenu and Damian Pérez Mazliah, PhD for their editorial assistance.

\section{REFERENCES}

1. Roundtable Discussion. The Ins and Outs of Incorporating Patient Centricity in Drug Development. Pharmaceutical Executive Magazine. parexel Website. https://www.parexel.com/ news-events-resources/blog/ins-and-outs-incorporating-patient-centricity-drug-development. Accessed November 15, 2019.

2. Barron D. Bridging the Diversity Gap in Clinical Trials. Web site. https://social.eyeforpharma.com/clinical/bridging-diversity-gap-clinical-trials. Accessed January 24, 2020.

3. Yeoman G, Furlong P, Seres M, Binder H, Chung H, Garzya V, et al. Defining patient centricity with patients for patients and caregivers: a collaborative endeavour. BMJ Innovations. 2017; 3(2):bmjinnov-2016-000157. doi: 10.1136/bmjinnov-2016-000157 
4. Merriam Webster Dictionnary. merriam Web site. https://www. merriam-webster.com/. Accessed April 23, 2020.

5. Limkakeng A, Phadtare A, Shah J, Vaghasia M, Wei DY, Shah A, et al. Willingness to participate in clinical trials among patients of Chinese heritage: A meta-synthesis. PLoS ONE. 2013; 8(1): e51328. doi: 10.1371/journal.pone.0051328

6. Hughson J, Woodward-Kron R, Parker A, Hajek J, Bresin A, Knoch U, et al. A review of approaches to improve participation of culturally and linguistically diverse populations in clinical trials. Trials. 2016; 17:263. doi: 10.1186/s13063-016-1384-3

7. Yancey AK, Ortega AN, Kumanyika SK. Effective recruitment and retention of minority research participants. Annu Rev Public Health. 2006; 27: 1-28. doi: 10.1146/annurev. publhealth.27.021405.102113

8. Huang B, De Vore D, Chirinos C, Wolf J, Low D, Willard-Grace $\mathrm{R}$, et al. Strategies for recruitment and retention of underrepresented populations with chronic obstructive pulmonary disease for a clinical trial. BMC Med Res Methodol. 2019; 19(1): 39. doi: 10.1186/ s12874-019-0679-y

9. Nasha B, Mishra M, Pentz R, Owonikoko TK. Enrollment of racial minorities in clinical trials: Old problem assumes new urgency in the age of immunotherapy. Am Soc Clin Oncol Educ Book. 2019; 39: 3-10. doi: 10.1200/EDBK_100021

10. Fisher JA, Kalbaugh CA. Challenging assumptions about minority participation in US clinical research. Am J Public Health. 2011; 101(12): 2217-2222. doi: 10.2105/AJPH.2011.300279

11. Food and Drug Administration, Society for Women's Health Research. Dialogues on Diversifying Clinical Trials. FDA Web site. https://www.fda.gov/media/84982/download. Accessed April 23, 2020 .

12. Quay TA, Frimer L, Janssen PA, Lamers Y. Barriers and facilitators to recruitment of South Asians to health research: A scoping review. BMJ Open. 2017; 7: e014889. doi: 10.1136/bmjopen-2016-014889

13. Food and Drug Administration. Enhancing the Diversity of Clinical Trial Populations - Eligibility Criteria, Enrollment Practices, and Trial Designs Guidance for Industry. FDA Web site. https://www.fda.gov/regulatory-information/search-fda-guidance-documents/enhancing-diversity-clinical-trial-populations-eligibility-criteria-enrollment-practices-and-trial. Accessed June 4 2019.

14. Liu KA, Di Pietro Mager NA. Women's involvement in clinical trials: historical perspective and future implications. Pharm Pract (Granada). 2016; 14(1): 708. doi: 10.18549/PharmPract.2016.01.708 15. Berlin JA, Ellenberg SS. Inclusion of women in clinical trials. BMC Med. 2009; 7: 56. doi: 10.1186/1741-7015-7-56
16. Ruiz Cantero MT, Pardo MA. European Medicines Agency policies for clinical trials leave women unprotected. J Epidemiol Community Health. 2006; 60: 911-913. doi: 10.1136/jech.2006.048769

17. Labots G, Jones A, de Visser SJ, Rissman R, Burggraaf J. Gender differences in clinical registration trials: Is there a real problem? Br J Clin Pharmacol. 2018; 84; 700-707. doi: 10.1111/bcp.13497

18. Rosenberg R, Barker KA, Anderson WF. Future distribution of multiple myeloma in the United States by sex, age, and race/ethnicity. Blood. 2015; 125(2): 410-412. doi: 10.1182/ blood-2014-10-609461

19. Food and Drug Administration. FDA Drug Trails Snapshots. fda Web site. https://www.fda.gov/drugs/drug-approvals-and-databases/drug-trials-snapshots. Accessed May 18, 2020.

20. Oh SS, Galanter J, Thakur N, Pino-Yanes M, Barcelo NE, White MJ, et al. Diversity in clinical and biomedical research: A promise yet to be fulfilled. PLoS Med. 2015; 12(12): e1001918. doi: 10.1371/journal.pmed.1001918

21. Knepper T, McLeod H. When will clinical trials finally reflect diversity? Nature. 2018; 557(7704): 157-159. doi: 10.1038/d41586018-05049-5

22. World Medical Association (WMA). Declaration of Helsinki Ethical principles for medical research involving human subjects. WMA Web site. https://www.wma.net/policies-post/wma-declaration-of-helsinki-ethical-principles-for-medical-research-involving-human-subjects/. Accessed April 23, 2020.

23. International Council for Harmonisation of Technical Requirements for Pharmaceuticals for Human Use (ICH). ICH Topic E9 Statistical Principles for Clinical Trials. ICH Web site. https:/ / database.ich.org/sites/default/files/E9_Guideline.pdf. 1998. Accessed April 23, 2020.

24. Fejerman L, Ahmadiyeh N, Hu D, Huntsman S, Beckman KB, Caswell JL, et al. Genome-wide association study of breast cancer in Latinas identifies novel protective variants on 6q25. Nat Commun. 2014; 5: 5260. doi: 10.1038/ncomms6260

25. Ranjit N, Siefert K, Padmanabhan V. Bisphenol-A and disparities in birth outcomes: a review and directions for future research. J Perinatol. 2010; 30: 2-9. doi: 10.1038/jp.2009.90

26. Chung W-H, Hung S-I, Hong H-S, Hsih M-S, Yang L-C, Ho H-C, et al. Medical genetics: A marker for Stevens-Johnson syndrome. Nature. 2004; 428: 486. doi: 10.1038/428486a

27. Gandhi M, Greenblatt RM, Bacchetti P, Jin C, Huang Y, Anastos $\mathrm{K}$, et al. A single-nucleotide polymorphism in CYP2B6 leads to $>3$-fold increases in efavirenz concentrations in plasma and hair among HIV-infected women. J Infect Dis. 2012; 206: 1453-1461. doi: $10.1093 /$ infdis/jis508 
28. Genovese G, Friedman DJ, Ross MD, Lecordier L. Association of trypanolytic ApoL1 variants with kidney disease in African Americans. Science. 2010; 329(5993): 841-845. doi: 10.1126/ science. 1193032

29. Lackland DT. Racial differences in hypertension: Implications for high blood pressure management. Am J Med Sci. 2014; 348(2): 135-138. doi: 10.1097/MAJ.0000000000000308

30. Gupta AK. Racial Differences in Hypertension: Implications for High Blood Pressure Management. Int J Prev Med. 2010; 1(4): 217-219.

31. Youngmee K, Pavlish C, Evangelista LS, Kopple JD, Phillips LR. Racial/ethnic differences in illness perceptions in minority patients undergoing maintenance hemodialysis. Nephrol Nurs J. 2012; 39(1): 39-49.

32. Otado J, Kwagyan J, Edwards D, Ukaegbu A, Rockcliffe F, Osafo N. Culturally competent strategies for recruitment and retention of African American populations into clinical trials. Clin Transl Sci. 2015; 8(5): 460-466. doi: 10.1111/cts.12285

33. Sharde CT. It's Not You, It's Me: Necessity of Including Cultural Factors in Clinical Research. Law \& Ineq: A Journal of Theory and Practice. 2012; 30(1): 179-209.

34. Galen G, Dohan D. Diversity of participants in clinical trials in an academic medical center: the role of the 'Good Study Patient'? Cancer. 2009; 115(3): 608-615. doi: 10.1002/cncr.24028

35. Treweek S, Pitkethly M, Cook J, Fraser C, Mitchell E, Sullivan $\mathrm{F}$, et al. Strategies to improve recruitment to randomised trials. Cochrane Database Syst Rev. 2018; 2(2): MR000013. doi: 10.1002/14651858.MR000013.pub6

36. Epstein S. The rise of 'recruitmentology': Clinical research, racial knowledge, and the politics of inclusion and difference. Soc Stud Sci. 2008; 38(5): 801-832. doi: 10.1177/0306312708091930 